\title{
Multinodular and Vacuolating Neuronal Tumor of the Cerebrum: Does the Name Require Review?
}

M ultinodular and vacuolating neuronal tumor (MVNT) of the cerebrum is a relatively novel entity added to the revised 2016 World Health Organization classification, ${ }^{1}$ though first described in 2013 . $^{2}$ It is characterized by a cluster of intra-axial nodules with mixed neuro-glial cells demonstrating internal vacuolating architecture. The lesions are benign and nonprogressive and, given their innocence, are a potential "no touch" or "leave-me-alone" lesion. ${ }^{3}$ All published cases have been described in the supratentorium. So far, there are no published cases of MVNT of the cerebellum. We have seen at least 3 cases with imaging characteristics identical to those of MVNT of the cerebrum that were observed in the cerebellum.

We had 3 patients between 23 and 39 years of age presenting with headaches ( 2 cases) and vertigo ( 1 case). The neurologic examination findings were unremarkable in all cases. MR imaging of the brain showed a cluster of nodules, 1-3 mm each, located in the superior vermis and within the surrounding superior cerebellar hemisphere (Figs 1-3). In all cases, the nodules were hypointense to gray matter on T1-weighted imaging, hyperintense on T2, and did not suppress completely on FLAIR imaging. There was no enhancement, susceptibility, or diffusion restriction. There was no surrounding edema or mass effect. The imaging features were consistent with the imaging descriptions of MVNT. A 12-month follow-up MR imaging showed no interval change.

We have no histopathologic evidence because these are "no touch" lesions. ${ }^{3,4}$ However, their exact imaging similarities to supratentorial MVNT and stability across time suggesting a benign natural history support that these likely represent the same histopathologic entity involving the cerebellum. We propose that the name of the entity be expanded to "MVNT of the brain" to include cerebellar lesions in the phenotypic spectrum of this newly described entity.

http://dx.doi.org/10.3174/ajnr.A6284
Disclosures: Aanchal Agarwal—UNRELATED: Employment: Sir Charles Gairdner Hospital.

\section{REFERENCES}

1. Louis DN, Perry A, Reifenberger G, et al. The 2016 World Health Organization Classification of Tumors of the Central Nervous System: a summary. Acta Neuropathol 2016;131:803-20 CrossRef Medline

2. Huse JT, Edgar M, Halliday J, et al. Multinodular and vacuolating neuronal tumors of the cerebrum: 10 cases of a distinctive seizure-associated lesion. Brain Pathol 2013;23:515-24 CrossRef Medline

3. Nunes RH, Hsu CC, da Rocha AJ, et al. Multinodular and vacuolating neuronal tumor of the cerebrum: a new "leave me alone" lesion with a characteristic imaging pattern. AJNR Am J Neuroradiol 2017; 38:1899-904 CrossRef Medline

4. Alsufayan R, Alcaide-Leon P, de Tilly LN, et al. Natural history of lesions with the MR imaging appearance of multinodular and vacuolating neuronal tumor. Neuroradiology 2017;59:873-83 CrossRef Medline

(D) A. Agarwal Neurointervention and Imaging Services of Western Australia Sir Charles Gairdner Hospital Nedlands, Western Australia

(1) R. Lakshmanan
Princess Margaret Hospital
Subiaco, Western Australia
University College of London Foundation Trust
National Hospital for Neurology and Neurosurgery, London
London, UK
University College of London Foundation Trust
National Hospital for Neurology and Neurosurgery, London
London, UK
Neurointervention and Imaging Services of Western Australia
Sir Charles Gairdner Hospital
Nedlands, Western Australia
Princess Margaret Hospital
Subiaco, Western Australia




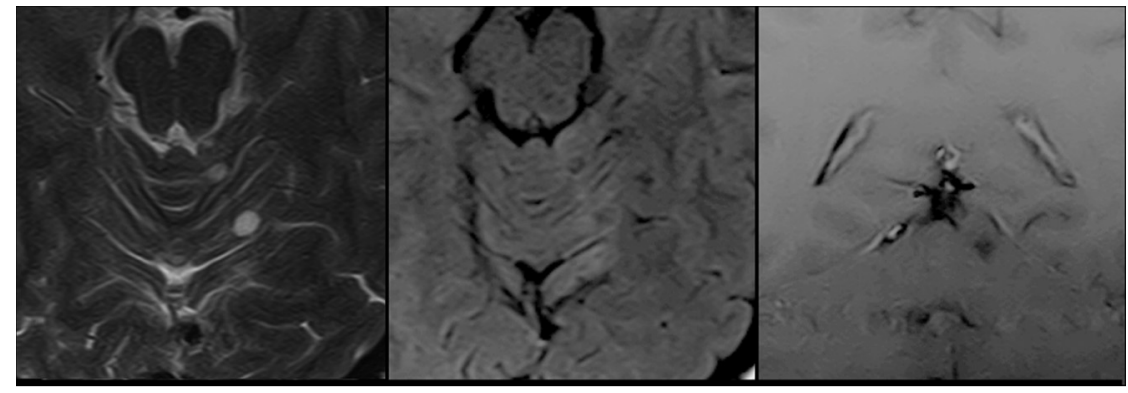

FIG 1. Montage of images (axial T2, axial FLAIR, coronal T1 fat-saturated postcontrast) shows at least 2 T2 hyperintense nodules of 3 and $5 \mathrm{~mm}$ each to the left of the superior vermis. These are hyperintense to CSF on T2, show incomplete suppression on FLAIR, and do not enhance.

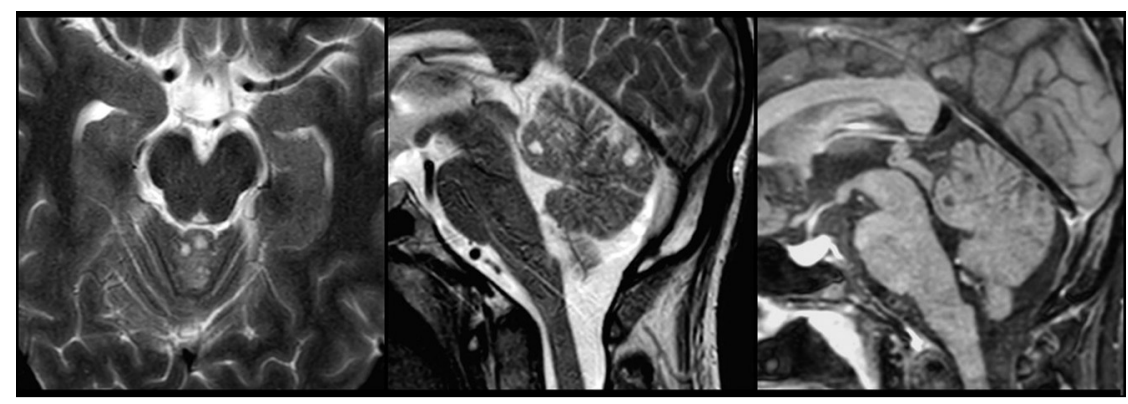

FIG 2. Montage of images (axial T2, sagittal T2, and sagittal T1 postcontrast) shows small T2 hyperintense nodules in the superior vermis. They do not enhance.

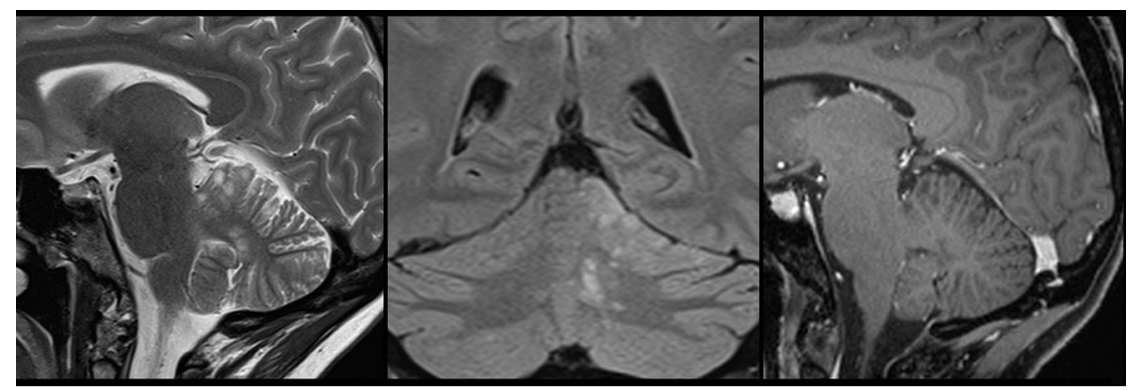

FIG 3. Montage of images (sagittal T2, coronal FLAIR, and sagittal Tl fat-saturated postcontrast) shows a cluster of small nodules of similar size within the left paravermian and cerebellar hemisphere. 